\title{
Lidil
}

Revue de linguistique et de didactique des langues

$50 \mid 2014$

Variation stylistique et diversité des contextes de socialisation

\section{Variation stylistique par alternance codique en contexte maltais}

Stylistic Variation through Code-Switching in the Maltese Context

\section{Anne-Marie Bezzina}

\section{(2) OpenEdition}

Journals

Édition électronique

URL : http://journals.openedition.org/lidil/3544

DOI : 10.4000/lidil.3544

ISSN : 1960-6052

Éditeur

UGA Éditions/Université Grenoble Alpes

Édition imprimée

Date de publication : 15 décembre 2014

Pagination : 17-43

ISBN : 978-2-84310-287-5

ISSN : $1146-6480$

\section{Référence électronique}

Anne-Marie Bezzina, "Variation stylistique par alternance codique en contexte maltais », Lidil [En

ligne], 50 | 2014, mis en ligne le 15 juin 2016, consulté le 19 avril 2019. URL : http://

journals.openedition.org/lidil/3544; DOI : 10.4000/lidil.3544

(c) Lidil 


\title{
Variation stylistique par alternance codique en contexte maltais
}

\author{
Anne-Marie Bezzina*
}

\begin{abstract}
RÉSUMÉ
Cette étude vise à interpréter les implications sociolinguistiques de l'alternance codique entre le maltais et l'anglais à partir de l'analyse d'un corpus de discours oral produit par des locuteurs maltais bilingues. Il s'en dégage des différences comportementales, au niveau de l'alternance, entre des locuteurs professionnels et non professionnels de la parole publique, et en fonction du contexte décontracté ou de formalité (protocolaire ou autre). L'anglais jouit d'un prestige marqué et peut servir d'instrument pour affirmer le propre statut social, le professionnalisme ou l'appartenance à un groupe plutôt qu'à un autre. Des changements au niveau de la configuration de la société maltaise favorisent le changement linguistique, et inversement.
\end{abstract}

\begin{abstract}
The aim of this study, which is based on a corpus of spoken discourse produced by bilingual Maltese speakers, is to describe some possible sociolinguistic implications of switching between Maltese and English in Malta. Public speech professionals and more ordinary speakers show different switching habits, and diverse patterns also emerge according to whether the context is relaxed or formal, be it institutional formality or more usual formality as in some transactional interactions. Socially, prestige is associated with English. This language can serve as an instrument to affirm one's social status and professional position, as well as to express identity through one's allegiance to a group's norms rather than those of another. Changes affecting the traditional configuration of Maltese society seem to favour language shift, and vice versa.
\end{abstract}

* Université de Malte. 


\section{Introduction}

Cette étude vise à observer le discours oral de locuteurs maltais du point de vue du changement stylistique, afin de dégager des schémas comportementaux communs ou particuliers au niveau de l'alternance codique. C'est le cadre bilingue de Malte ${ }^{1}$ qui permet à la variation stylistique de se réaliser à travers l'alternance codique entre le maltais et l'anglais. Dans la recherche, à l'instar de Gumperz (1982), il a souvent été postulé une identité fonctionnelle entre la variation de registre en contexte monolingue et la variation réalisée à travers l'alternance codique en situation bilingue. Les chercheurs tendent à associer la variation passant par le registre aux locuteurs monolingues et la variation par alternance codique aux locuteurs bilingues (Bell, 2001; Gadet, 2005; Traugott \& Romaine, 1985). Nous avons ailleurs établi qu'il existe bien au sein de la langue maltaise une variation stylistique passant par le registre, et donc que dans le cadre maltais, les deux formes de manifestation de la variation stylistique coexistent et sont réalisées par les mêmes locuteurs - tous bilingues (Busuttil Bezzina, 2013). L'attention sera ici uniquement portée sur la variation stylistique réalisée par alternance codique.

La présente analyse concerne le va-et-vient d'une langue à l'autre, dans le sens du mélange intraphrastique du maltais et de l'anglais. Nous utilisons par commodité le terme de «alternance codique» pour ce phénomène, tout en précisant que notre approche ne se focalise pas sur l'aspect linguistique de l'alternance : ce qui nous intéresse, ce sont les significations sociales véhiculées par le mélange et les négociations interpersonnelles contractées entre les interactants plurilingues dans leur recours à l'alternance, qui peut être axé sur l'accentuation autant des similitudes et de l'inclusion, que des distinctions et de l'exclusion. Nous nous approchons, par cette visée fondée sur les fonctions plutôt que sur la forme, des positions théoriques autour du concept de translanguaging, défini comme «the process of making meaning [and]

1. On parle de Malte comme pays bilingue, mais, dans les îles, à part le maltais standard et l'anglais, certains parlent le maltais dialectal (surtout réservé à des usages informels dans les zones rurales) et une variété appelée par les linguistes le mélange maltais-anglais (Borg, 1980), fondée sur un va-et-vient systématique (mais qui ne répond à aucune règle formelle) entre le maltais et l'anglais. 
shaping experiences [...] through the use of two languages ${ }^{2} »$ (Baker, Jones \& Lewis, 2012, p. 1). Il sera pourtant ici fait abstraction des usages fréquents de cette théorie dans le cadre de l'éducation scolaire. L'association fréquente du translanguaging avec les classes plurilingues est en effet l'une des raisons pour lesquelles nous préférons utiliser le terme de «alternance codique». Translanguaging a été traduit en français comme «trans-apprentissage linguistique» (Rehorick, 2009), alors que notre étude porte sur la vie de locuteurs bilingues en dehors du secteur de l'éducation. Nous ne souscrivons pas, pour autant, à la conception qui peut être connotée par le terme de «alternance codique», que les langues soient des entités discrètes; dans les cadres plurilingues, on atteste des pratiques souples et entrelacées. Garcia utilise l'expression «fuzziness of the language practices» («le flou des pratiques langagières», 2009, p. 150-151) pour ces cadres. Mise à part la terminologie, le concept de translanguaging s'adapte mieux à notre visée, car il va au-delà de ce que le terme de «alternance codique» laisse traditionnellement entendre avec sa mise en relief du coté linguistique : «Translanguaging [...] goes beyond what has been termed codeswitching, although it includes $i t^{3} \gg$ (Garcia, 2009, p. 140). Le comportement des locuteurs informateurs de notre corpus nous amène à prendre du recul également par rapport à une interprétation assez répandue des motivations du translanguaging: "Translanguaging is the act performed by bilinguals of accessing different linguistic features or various modes of what are described as autonomous languages, in order to maximize communicative potential ${ }^{4} \gg($ Garcia, 2009, p. 140 ; c'est nous qui soulignons). Dans le cadre maltais et certainement pour ce qui est des locuteurs de notre corpus, tous les individus partagent le même bagage linguistique, et l'alternance ne se fait pas pour permettre la compréhension et la communication efficace, mais plutôt pour transmettre des valeurs sociolinguistiques spécifiques en puisant dans les valeurs associées à des styles différents.

2. Notre traduction : «le processus de créer du sens [et] de constituer des expériences $[\ldots]$ à travers l'usage de deux langues ».

3. Notre traduction : «Le translanguaging [...] va au-delà de ce qu'on a appelé "alternance codique", bien qu'il l'inclue».

4. Notre traduction : «Le translanguaging est l'acte réalisé par les individus qui accèdent à différents traits linguistiques ou à des modes variés de ce qu'on décrit comme des langues autonomes, afin de favoriser le potentiel communicatif.» 
Une large gamme de contingences situationnelles conditionnent les changements stylistiques : la formalité de la situation de communication, la composition de l'audience, le sujet, le cadre de la situation, le ton (sérieux ou de plaisanterie), le medium (écrit ou oral), mais aussi le contexte co-construit et reconstruit par les participants à l'échange tout au long de l'interaction, leurs objectifs et les enjeux identitaires (Busuttil Bezzina, 2013; Gadet, 2007). Alors que les premières études sociolinguistiques (dont Labov, 1972) interprétaient la variation stylistique uniquement en termes d'effets de la situation sur le discours (le discours étant tenu comme reflet des données situationnelles ${ }^{5}$ ), les analyses plus récentes font une large part à l'initiative des locuteurs, et à leur créativité (intentionnelle) du style au niveau de l'investissement identitaire (Coupland, 2001 ; Eckert, 2000 ; Schilling-Estes, 2002). Ceci fait que les changements de style ne sont pas prévisibles, mais interprétables (Bell, 1984), par la prise en compte de l'influence réciproque qu'exercent les uns sur les autres les facteurs situationnels, discursifs et pragmatiques.

\section{Bilinguisme et diglossie en contexte maltais}

Afin de mieux pouvoir interpréter les implications sociolinguistiques du changement stylistique en contexte maltais, il est nécessaire de comprendre la configuration linguistique qui caractérise cet archipel minuscule situé à $96 \mathrm{~km}$ au sud de la Sicile (population : 420000). Ancienne colonie britannique, Malte a une langue nationale, le maltais, et deux langues officielles, le maltais et l'anglais. Le maltais est la principale langue de communication orale. Dans les zones branchées de l'île, une partie des habitants n'utilise que l'anglais ou le mélange maltais-anglais ${ }^{6}$ (désormais MMA). Le reste de la population les appelle par moquerie tal-pepè (les «fins snobs» de la ville de Sliema et de sa région) pour leur façon de parler. Certaines zones ont des dialectes régionaux, que beaucoup voient d'un mauvais oil en les appelant talћamalli (des «criards»); ces dialectes sont abandonnés au profit du

5. Voir pour une critique Gadet (1998).

6. Il faut clairement établir une distinction entre le mélange maltais-anglais, dont l'alternance omniprésente est la principale caractéristique, et le discours en maltais, qui ne contient que sporadiquement des termes ou expressions anglais (voir la note 1). 
maltais standard dès qu'un locuteur parle à un inconnu ou dans une situation formelle. Certains locuteurs d'anglais L1 méprisent le maltais pour son utilité limitée, et ses locuteurs sont perçus comme des individus ayant un niveau d'éducation inférieur. Une enquête par questionnaire que nous avons menée en 2008, portant sur l'emploi des différentes variétés et les attitudes linguistiques, confirme la tension qui caractérise la situation linguistique à Malte : la question des variétés éveille des sentiments vifs, exprimés par un langage qui fait ressortir l'antipathie des uns et des autres envers telle ou telle catégorie de locuteurs ${ }^{7}$ (Busuttil Bezzina, 2013).

Certains chercheurs estiment que le bilinguisme de Malte n'admet pas de rapport diglossique entre l'anglais et le maltais (Borg, 1980; Camilleri, 1995), en se basant sur la différentiation partielle des fonctions remplies par les deux langues. En fait, il existe des schémas distributionnels nets, et l'une ou l'autre langue domine chaque secteur d'activité important (Busuttil Bezzina, 2013). Le maltais domine au niveau du pouvoir juridique, de la fonction publique et des médias. Pourtant, en dehors de ces cadres institutionnels où le maltais est imposé, c'est l'anglais qui jouit du statut de variété High (désormais $\mathrm{H}$ ). Le maltais est de loin la langue la plus utilisée pour la conversation quotidienne, mais la majorité de la population écrit automatiquement en anglais ${ }^{8}$. Les communications écrites du secteur privé (entreprises, institutions financières, organisations diverses) et les pancartes dans les magasins sont en anglais. Le maltais n'est pas assez développé lexicalement pour qu'on s'en serve dans certains secteurs professionnels, comme la médecine, l'ingénierie et l'informatique. L'anglais est la langue des colloques, de l'éducation universitaire et de la recherche.

7. Ainsi, les sondés, majoritairement locuteurs du maltais L1, ont massivement exprimé leur condamnation de l'alternance codique (malgré son omniprésence), des locuteurs d'anglais L1 surtout lorsqu'ils n'ont que de faibles compétences d'anglais, des parents maltophones qui éduquent leurs enfants à parler l'anglais comme L1, etc. La stigmatisation du maltais dialectal est également ressortie, tout comme un certain sentiment de supériorité des quelques sondés locuteurs d'anglais L1.

8. Faute d'études, on ne peut pas se prononcer sur les motifs : défaut de compétence ou incertitude face aux complications du maltais (et ses graphèmes non prononcés), habitude presque généralisée, volonté de démontrer des compétences d'anglais? 
Les spots publicitaires sont dans l'une ou l'autre langue, en fonction du type de public visé.

En fait, même les chercheurs qui soulignent l'avancement du maltais et partant l'absence de diglossie, ne manquent pas de parler des fonctions différentes remplies par chacune des langues, ou du prestige majeur dont jouit l'anglais : «English serves the function of a High variety and Standard serves as the Low variety, with the possibility however that it may also complement English in some of its High functions ${ }^{9}$.» (Borg, 1980, p. 9) L'emploi répandu du MMA constitue une preuve du prestige associé à l'anglais.

À notre avis, l'affirmation que la situation linguistique de Malte se caractérise par le bilinguisme sans diglossie ne peut s'appliquer qu'au niveau institutionnel ${ }^{10}$, où la projection du maltais est forte. Au niveau social, la diglossie régit les rapports entre les deux langues. Il serait plus pertinent de décrire la communauté maltaise comme relativement diglossique (Busuttil Bezzina, 2013). Le recouvrement des deux langues dans certains domaines représente des changements en cours (Romaine, 1995), sur lesquels nous reviendrons plus loin.

\section{Le corpus}

Afin d'analyser la variation intralocuteur, quatre locuteurs-clés ont été enregistrés, avec leur accord préalable, dans plusieurs situations marquées par différents degrés de formalité. Le choix des locuteurs se basait sur notre postulat qu'il existe en maltais (comme dans d'autres langues) deux types distincts de discours formels, que nous avons appelés «le formel protocolaire» et «le formel des situations non institutionnelles». Le premier serait appris par l'éducation formelle et pratiqué par des professionnels de la parole publique dans des situations à enjeu «sérieux» ou médiatiques; le second serait pratiqué par tous, normalement dans des cadres transactionnels, souvent dans des échanges avec des experts. Afin de mettre en place les conditions pour pouvoir tester cette hypo-

9. Notre traduction : «L'anglais sert la fonction de variété $H$ et le maltais standard celle de variété Low (désormais L), avec pourtant la possibilité qu'il remplisse aussi des fonctions $\mathrm{H}$.»

10. Dans ce cas on doit faire exclusion de l'institution éducative, qui admet des rapports diglossiques entre les deux langues, l'anglais étant le principal véhicule de l'enseignement (Caruana, 2011). 
thèse, nous nous sommes inspirée de Bilger et Cappeau (2004), qui suivent eux aussi des locuteurs dans des corpus longs pour identifier selon quelles modalités un même locuteur utilise différents styles. Les auteurs analysent le discours d'un locuteur non professionnel de la parole publique et celui d'un homme politique, afin de voir comment la notion de registre permet d'expliquer les différences entre leurs discours. Nous avions parmi nos locuteurs deux professionnels de la parole publique (le vice-Premier ministre et un professeur universitaire) et deux locuteurs non professionnels (un cadre, une assistante en pharmacie). À part le vice-Premier ministre (désormais vice-PM), dont nous avons fait connaissance préalablement aux enregistrements, les autres participants étaient des personnes antérieurement connues, ce qui nous a permis de les suivre dans diverses situations en les y accompagnant. Ces personnes font partie d'un réseau familial, ce qui offre l'avantage de faire émerger, plus que dans les interactions entre inconnus, des schémas discursifs particuliers comme l'ironie, l'allusion, et les présuppositions non explicitées (Tannen, 2005).

L'enregistrement des interactions PARLEMENT (vice-PM) et RADIO (professeur), qui nous ont été signalées par les locuteurs euxmêmes, était disponible sur l'Internet. Nous avons laissé notre appareil digital au vice-PM et au cadre pour qu'ils enregistrent des sessions dans leurs bureaux respectifs. Dans tous les autres cas, nous étions présente et avons nous-même effectué les enregistrements. Avec leur accord préalable, les personnes n'étaient souvent pas au courant de la présence ou du fonctionnement de l'appareil. Le tableau 1 indique le participant principal et la taille de chacune des interactions, le lieu où elle s'est déroulée et la situation d'interaction. Le corpus oral transcrit (par nousmême) totalise plus de 60000 mots. Nous avons analysé les interactions pour les occurrences d'alternance. 


\begin{tabular}{|c|c|c|}
\hline \multicolumn{3}{|c|}{ RÉPARTITION DES INTERACTIONS DANS L'ÉCHANTILLON } \\
\hline & \multicolumn{2}{|c|}{ PROFESSIONNELS DE LA PAROLE PUBLIQUE } \\
\hline Situation & $\begin{array}{c}\text { PROFESSEUR } \\
\text { UNIVERSITAIRE } \\
\end{array}$ & VICE-PREMIER MINISTRE \\
\hline formelle & $\begin{array}{l}\text { Émission radiophonique portant } \\
\text { sur son expertise d'historien - } \\
\text { RADIO a } \\
\text { studio } ; 27 \text { minutes }\end{array}$ & $\begin{array}{l}\text { Intervention parlementaire - } \\
\text { PARLEMENT } \\
\text { parlement } ; 34 \text { minutes }\end{array}$ \\
\hline semi-formelle $^{b}$ & & $\begin{array}{l}\text { Réunion avec son assistant } \\
\text { personnel - ASSISTANT } \\
\text { bureau du vice-PM; } 11 \text { minutes }\end{array}$ \\
\hline \multirow[t]{3}{*}{ informelle } & $\begin{array}{l}\text { 1. Fête de première communion } \\
\text { de sa nièce - FÊTE } \\
\text { chez son beau-frère; } 24 \text { minutes } \\
\text { 2. Diner chez lui ; la famille de sa } \\
\text { belle-sœur est invitée - DINER } \\
\text { salon/salle à manger; } 65 \text { minutes }\end{array}$ & \\
\hline & \multicolumn{2}{|c|}{ NON-PROFESSIONNELS DE LA PAROLE PUBLIQUE } \\
\hline & ASSISTANTE EN PHARMACIE & CADRE \\
\hline formelle & $\begin{array}{l}\text { Rendez-vous avec un agent } \\
\text { financier sur l'héritage du père / des } \\
\text { investissements - } \\
\text { COMPAGNIE FINANCIÈRE } \\
\text { bureau de la compagnie; } \\
20 \text { minutes }\end{array}$ & \\
\hline semi-formelle & $\begin{array}{l}\text { Vaccination de sa sœur et de ses } \\
\text { neveux - VACCIN } \\
\text { clinique publique où elle travaille; } \\
20 \text { minutes }\end{array}$ & $\begin{array}{l}\text { Session de planification du } \\
\text { travail avec son supérieur - } \\
\text { PLANIFICATION } \\
\text { bureau de l'entreprise; } 32 \text { minutes }\end{array}$ \\
\hline informelle & $\begin{array}{l}\text { 1. Diner chez elle }: \text { la famille de sa } \\
\text { sœur est invitée - DINER } \\
\text { salon/salle à manger } ; 65 \text { minutes } \\
\text { 2. Fête de première communion de } \\
\text { sa nièce - FÊTE } \\
\text { chez son frère } ; 24 \text { minutes } \\
\text { 3. Déjeuner en famille - } \\
\text { RESTAURANT } \\
\text { au restaurant } ; 15 \text { minutes }\end{array}$ & $\begin{array}{l}\text { 1. Planification d'un décernement } \\
\text { des prix avec son supérieur - PRIX } \\
\text { bureau de l'entreprise } ; 3 \text { minutes } \\
\text { 2. Fête de première communion de } \\
\text { sa nièce - FÊTE } \\
\text { chez son beau-frère } ; 24 \text { minutes } \\
\text { 3. Diner chez sa belle-sœur - } \\
\text { DINER } \\
\text { salon/salle à manger } ; 65 \text { minutes }\end{array}$ \\
\hline
\end{tabular}

a. Les mots en majuscules indiquent le code attribué à chaque interaction; le code sera utilisé dans les exemples pour renvoyer à telle ou telle interaction.

b. La catégorie des interactions «semi-formelles» ou «semi-informelles» correspond à ces interactions dont certains éléments les rapprochent du formel (par exemple, la distance entre interlocuteurs, le cadre spatio-temporel, le médium, et ainsi de suite) et d'autres de l'informel (par exemple, un sujet plutôt banal ou personnel). Ces classifications concernant le degré de formalité des interactions ne sont qu'approximatives, puisqu'elles se basent sur notre jugement intuitif selon le genre discursif et le contexte communicationnel. Elles doivent simplement servir de point de départ pour permettre une organisation pratique du corpus, et ne sont pas un résultat définitif de l'analyse de l'échantillon discursif.

Tableau 1. - Répartition des interactions du corpus selon les locuteurs. 


\section{L'alternance codique en fonction du style et du locuteur}

La division entre locuteurs professionnels et non professionnels de la parole publique a des retombées importantes sur les schémas variationnels manifestés en fonction du type et du degré de formalité des situations de communication. L'analyse du corpus suggère que dans le cadre maltais, l'alternance codique est l'une des principales manifestations de la différence entre formel protocolaire et formel des situations non institutionnelles. Dans les contextes institutionnels du parlement et de la radio, où le maltais est formellement imposé par les politiques linguistiques officielles, les deux professionnels de la parole publique tâchent d'éviter l'alternance. Ainsi, dans le texte RADIO, où le professeur est interviewé pour son expertise d'historien ${ }^{11}$, le recours à l'anglais est minimal et se limite aux cas où le locuteur est en mal de mot, ce que l'on remarque à travers l'hésitation dans l'exemple 1. Le présentateur de l'émission lui souffle alors le mot maltais ${ }^{12}$ :

L2 : le assolutament / inti

CITTADIN / fil-post fej= ghandek il-*belongings* haw $=$ eh:::

L1 : id-domicilju tieghak ${ }^{13}$

PROFESSEUR - RADIO
L2 : non absolument / tu es CITOYEN / à l'endroit où tu possèdes tes *belongings* (possessions) quoi euh :::

L1 : ton domicile

Cette insistance à utiliser le mot maltais se comprend, compte tenu des règlements sur le bon usage de la langue maltaise dans les médias et les sanctions encourues (sous forme d'amendes). Une fois libre des contraintes institutionnelles, le professeur recourt pourtant souvent à

11. Il s'agit d'une émission de la radio de l'université, destinée à un public éduqué. Le professeur publie en anglais, mais les règlements officiels sur l'emploi du maltais dans les médias imposent un maltais «correct».

12. Pour les conventions de transcription, voir le tableau en annexe.

13. Les extraits transcrits reproduisent les prononciations non standard qui tendent à indiquer de l'hypercorrection : le locuteur a ainsi ici prononcé tieghak pour tieghek. 
l'alternance codique jusque dans ses interactions informelles, lorsqu'il s'agit par exemple de termes techniques ${ }^{14}$ :

L6 : < le sujet est le musée des Invalides $>$ in-*Napoleonic Wars* [...] sas-*second world war* / kemm trid li rajt / imma fl-aћћar (tigba' hu=?) [...] ghax huma kienu jispećcjaliżżaw / specjjalment fil-*Hundred Years War* / kienu jispećjaliżżaw filkavallerija /// ara 1-Ingliżi kellhom il-*common soldiers* haw $=$ il(1-irg̉iel jimxu?)

\section{PROFESSEUR - DINER}

\begin{abstract}
L6 : les *Napoleonic Wars* (guerres napoléoniennes) $[\ldots]$ jusqu'à la *second world war* (deuxième guerre mondiale) / j'ai vu énormément de choses / mais finalement tu en as marre hein? [...] parce que eux ils se spécialisaient / surtout durant la *Hundred Years War* (Guerre des Cent Ans) / ils se spécialisaient en cavalerie /// par contre les Anglais avaient les *common soldiers* $($ fantassins $)=$ fin $($ les hommes qui marchent?)
\end{abstract}

Ce qui expliquerait ce comportement verbal du professeur pourrait être soit l'habitude d'employer ce genre de terme en anglais, soit la volonté d'affirmer son statut d'expert par le recours à l'anglais. Cette dernière possibilité est d'autant plus plausible que l'alternance ne se limite pas, chez lui, aux termes techniques, mais va notamment de pair avec l'acte de l'évaluation, lorsqu'il pose des jugements sur une multitude de sujets en invoquant (de manière implicite) son statut de personne instruite : au moins treize occurrences de jugements partiellement formulés en anglais ont été repérées dans ses interactions informelles (dont l'exemple 3) :

L6 : < le sujet est ses vacances à Paris $>*$ it was wise* $[\ldots] *$ it was very good* $[\ldots]$ veru sabiha /

*it's a bit complicated* eh?

PROFESSEUR - DINER
L6 : *it was wise* (c'était sage) $[\ldots] *$ it was very good* (c'était très bien) $[\ldots]$ elle est vraiment belle / *it's a bit complicated* (c'est un peu compliqué) hein?

14. Les termes techniques de l'histoire existent en maltais, mais la recherche académique se faisant le plus souvent en anglais, il est plus naturel pour le professeur de les produire en cette langue. 


\section{Enjeux identitaires}

L'usage de l'anglais souligne l'appartenance du professeur à l'élite académique et intellectuelle. Le cas de M. Tonio Borg, vice-PM, est particulièrement révélateur à cet égard : le maltais employé par ce professionnel de la parole publique dans un contexte institutionnel et formel, cède vite la place au prestige de l'anglais, au moins par l'usage du MMA, lorsque ce personnage parle avec son assistant dans un cadre moins formel et non protocolaire à la fin de la journée de travail. On a vu plus haut que la recherche en translanguaging souligne la souplesse qui caractérise normalement les changements de langue dans la plupart des cadres plurilingues : les frontières entre langues sont floues et permettent la fluidité dans les pratiques linguistiques et dans la construction des identités linguistiques (Garcia, 2009). Dans la contribution du vice-PM, ce genre de mouvement naturel d'une langue à l'autre, au point que les langues paraissent ne plus avoir de frontières nettes, se limite à son interaction avec son assistant. Dans son intervention parlementaire, il laisse voir une intention claire d'éviter l'alternance autant que possible (voir les exemples ci-dessous). Même s'il n'y réussit pas tout à fait, le taux de termes anglais est minime ${ }^{15}$, comparé à l'investissement lexical en maltais. La séparation codique dans ce contexte est plutôt comparable à celle d'une série de discours formels, destinés à être lus à différents publics ${ }^{16}$, qu'il a rédigés exclusivement en maltais. L'intervention parlementaire, bien que de nature beaucoup plus spontanée car les points sont développés sur-le-champ, révèle elle aussi un grand effort pour utiliser le maltais, qui y est la langue privilégiée même pour les termes techniques (alors que les locuteurs maltais les réalisent d'habitude en anglais dans des contextes non protocolaires). Il en va de même pour des termes qui sont, par conformité à des attitudes de

15. On trouve dans le texte PARLEMENT (6467 mots), 259 mots en anglais (4\%), dont beaucoup sont d'ailleurs des répétitions des mêmes termes techniques (12 fois les 2 mots Mister Speaker, 8 fois les 2 mots select committee, etc.), pour certains desquels il n'existe pas vraiment de terme maltais (36 fois pairing, 18 fois ruling, etc.). Par contre, on trouve 249 items lexicaux anglais dans le texte Assistant (2280 mots), soit $11 \%$ du nombre total des mots, et ces items correspondent souvent à des termes communs, non techniques, et variés.

16. Il s'agit d'un discours pour la Journée de la femme, d'un discours aux maires de l'Est, et d'un discours aux diplomates. 
valorisation de l'anglais, susceptibles d'être réalisés dans cette langue selon une «mode» plutôt moderne, comme les noms des jours de la semaine, des mois et des dates (Busuttil Bezzina, 2013), et pour les chiffres, alors que ceux-ci sont à Malte très souvent réalisés en anglais (Cucciardi, 1990).

Ainsi, si dans l'intervention parlementaire on trouve des termes techniques en maltais, comme tal-Kunsill tal-Ewropa (du Conseil de l'Europe), il-mozzjoni proćedurali (la motion de procédure), ce genre de termes sont systématiquement réalisés en anglais dans l'interaction avec l'assistant : rridu niddiskutuha fil-*United Nations* / fis-*Security Council* (nous devons en discuter dans les *United Nations* (Nations unies)/ dans le *Security Council* (Conseil de sécurité). Et alors qu'avec son assistant, il réalise les noms des jours de la semaine en anglais (exemple 4), il les énonce en maltais au parlement (exemple 5) :

tridu ssibu data [...] * preferably

Thursday / preferably*

L2: *a / a Thursday*?

L1 : *Thursday* jew *Friday*

VICE-PM (L1) - ASSISTANT

(5)

L2 : [...] ma jittihidx il-vot propju dikinhar / imma jittieћed 1-Erbgha filghodu [...] = igifieri jekk jin- jintalab it-Tnejn ...

VICE-PM - PARLEMENT vous devez identifier une date [...] * preferably Thursday / preferably* (de préférence par un jeudi / de préférence)

L2 : *a / a Thursday*? (un / un jeudi)

L1 : *Thursday* (jeudi) ou

*Friday* (vendredi)

L2 : [...] le vote ne soit pas pris ce jour-là / mais il soit pris le mercredi au matin [...] c'est-àdire que s'il est dem- demandé par un lundi ...

Dans les deux textes en question, les dates sont en distribution complémentaire, en anglais dans le texte ASSISTANT : mela llum gћandna *ninth* (alors aujourd'hui on est le *ninth* (neuf), et en maltais dans le texte PARLEMENT : il-verdett tal-poplu / tat-tmienja ta' Marzu (le verdict du peuple / du huit mars). Il y a également une distribution complémentaire des horaires en anglais dans le texte ASSISTANT (dont l'exemple 6) et en maltais dans le texte PARLEMENT (dont l'exemple 7) : 
(6)

hekk *about six thirty* per eżempju jew *seven*

VICE-PM - ASSISTANT

(7)

L2 : is-sedje qalet le / niћduh issa / fid-disgћa / neqsin hames minuti comme ça *about six thirty* (autour de dix-huit heures trente) par exemple ou *seven* (dix-neuf heures)

L2 : la présidence a dit non / on le prend maintenant / à vingt-etune heures / moins cinq minutes

\section{VICE-PM - PARLEMENT}

La comparaison entre textes n'est pas possible pour les années et les chiffres, qui ne figurent que dans le texte PARLEMENT où ils sont systématiquement en maltais ${ }^{17}$, comme dans dal-parlement / ghaddew minnu SBATAX -IL ELF / u seba' mitt domanda / parlamentari (dans ce parlement / il a été traité de dix-sept mille / sept cent questions / parlementaires). Les seules exceptions sont deux références à des années en anglais, dont : =ed TIGDEB / fin-*nineteen ninety-six* / niftakar / laqgha tal-grupp parlamentari / [...] u kont JIEN wiehed minn dawk li nsistejt / li NAGHTU l-*pairing* (vous MENTEZ / en *nineteen ninety-six* (mille neuf cent quatre-vingt-seize) / je me rappelle / une réunion du groupe parlementaire / [...] et j'étais MOI un de ceux qui ai insisté / de DONNER le pairage). Cette référence à une année en anglais remplit une fonction pragmatique : l'alternance attire l'attention sur les intentions communicatives du locuteur qui met en avant le contraste entre, d'une part, son parti qui avait souhaité, lorsqu'il était parti de l'opposition, accorder le pairage au parlement, et, d'autre part, l'actuel parti de l'opposition qu'il accuse de refuser d'accorder le pairage à son gouvernement pendant la législation courante. Les deux références à des années en anglais contrastent toutefois dans ce texte émis dans un cadre protocolaire avec non moins de treize occurrences de références en maltais :

17. Les années et les chiffres sont très couramment réalisés en anglais dans la conversation spontanée et même au-delà de ce genre de contexte informel. 
(8)

L2 : ma giet risposta ta' xejn /

elfejn u hamsa / elfejn u sitta /

elfejn u sebgha / elfejn u

TMIENJA / u ma giet l-ebda

risposta aucune réponse ne nous parvint / deux mille cinq / deux mille six / deux mille sept / deux mille HUIT / et aucune réponse ne nous parvint

\section{VICE-PM - PARLEMENT}

Lorsque le vice-PM interagit avec son assistant, les termes anglais qu'il utilise ne se limitent plus aux catégories susceptibles à l'alternance, comme les termes techniques, les noms des jours et des mois et les chiffres. L'alternance est dans ce cadre fréquemment fortuite :

(9)

$\begin{array}{ll}\text { imma:: jibdew jaћsbu politi- } & \text { mais:: qu'ils commencent à } \\ \text { kament / x’tip ta' programm } & \text { penser politiquement / à quel } \\ \text { jagћmlulu [...] imma: = gifieri: / } & \text { type de programme lui préparer } \\ \text { *they they have to own it* / } & {[\ldots] \text { mais: c'est-à-dire / *they }} \\ \text { fhimt? } & \text { they have to own it* (ils ils } \\ \text { VICE-PM - ASSISTANT } & \text { doivent en assumer la responsa- } \\ & \text { bilité) / tu comprends? }\end{array}$

Ces schémas divergents d'emploi des codes montrent qu'un locuteur professionnel de la parole publique en maltais formel peut immédiatement pencher vers le MMA une fois libéré de la contrainte de l'emploi institutionnel du maltais. Il parait que dans les situations de discours oral improvisé, marquées par le formel protocolaire, les frontières entre langues deviennent, sinon étanches, au moins bien plus épaisses que dans les situations qui admettent davantage de spontanéité.

Le locuteur, en adoptant un style protocolaire, ou bien un style plus libre contenant davantage d'alternance, peut être comparé à ces acteurs du théâtre grec de l'Antiquité qui changeaient de masque pour manifester différents états d'âme. Le locuteur se construit une identité en fonction de la présence de règles protocolaires strictes ou de leur relâchement. Il endosse lorsqu'il le faut une identité de vice-PM s'adressant à ses collègues parlementaires et au grand public du haut de sa position d'autorité en tant que numéro deux du gouvernement et leader parlementaire du parti au pouvoir, avec les obligations (même linguistiques) qu'un tel statut comporte. En dehors de ces cadres réglementés, l'inclusion de nombreux termes et expressions anglais dans le discours professionnel semble être associée à un certain statut, à l'efficacité, au 
professionnalisme, et dans certains cas, à l'autorité du décideur. Ceci va dans le sens des idées avancées par Canagarajah (1995) sur le pouvoir et la domination exercés par la langue anglaise à l'époque contemporaine, comme produit et vecteur de la mondialisation («global English»). En contexte maltais, l'anglais n'est pas nécessaire pour servir de lingua franca, mais il peut être exploité à des fins de contrôle social.

Ce dernier type de comportement verbal variable au niveau de l'alternance peut aussi être observé chez les locuteurs qui ne sont pas des figures publiques. L'assistante en pharmacie, femme du professeur, intègre l'anglais à divers degrés dans ses propos selon les situations dans lesquelles elle se trouve. Au restaurant, où la famille s'est réunie, les femmes ${ }^{18}$ parlent mode, famille et travail. À des moments précis, le changement de code effectué par l'assistante en pharmacie reflète un changement de footing ${ }^{19}$. Ainsi, de ses propos en maltais langue dominante, elle passe à une séquence en MMA, lorsqu'elle rapporte une conversation avec le médecin-chef, directeur général de la grande clinique publique où elle travaille, avec qui elle a «flirté» en anglais :

L3 : XXX issa m'in- / issa m'iniex nagћlqu $/ \mathrm{konn}==\mathrm{ed}$ nixorbu l-wiski / l-ieћor kien $=$ ed isajjar $/ / /$ eh jiena $1-* \mathrm{SMO} *$ stess qalli = gifieri dil-gimg dhalt ghandu ghidtlu aћћ *how nice* ghidtlu *your your office* / $\mathrm{da}=1-*$ head $^{*}$ tal-*clinic* $/$ l-*SMO* / ghidtlu / bil-*heating* u hekk / ghidtlu mhux bћal dawk 1-imgienen li ghandi hemm isfel ghidtlu bl-erkondixins ghaddejjin / qalli / *put down /

\author{
L3 : XXX maintenant on ne / \\ maintenant on ferme plus / \\ on était en train de boire du \\ whisky / l'autre il était en train \\ de cuisiner /// et puis moi c'est \\ le *SMO* (médecin-chef) lui- \\ même qui m'a dit après tout cette \\ semaine / je suis entrée chez \\ lui je lui ai dit ah:: *how nice* \\ (qu'est-ce que c'est bien) je lui \\ ai dit *your your office* (votre \\ votre bureau) / c'est le *head* \\ (chef) de la *clinic* (clinique)/
}

18. Il s'agit de l'assistante en pharmacie, sa sœur, infirmière, son autre sœur, enseignante, sa belle-sœur, femme au foyer, sa mère, institutrice retraitée, sa tante, ouvrière retraitée, et deux nièces, adolescentes.

19. La notion de footing (Goffman, 1981) se réfère à l'ajustement réalisé par les participants à une interaction, aux mouvements qui impliquent un changement de ton ainsi que de rôle social de la part des participants, comme un changement de vitesse qui a lieu chaque fois qu'on passe du transactionnel au banal ou vice versa. 
a small tot of whisky* mal-

*coffee* / tani hu biex naghmel

il-wiski hu? / *go ahead*

$<$ rires $>$

L4 : 1-*SMO* tal-*pharmacy* jigi jew?

L3 : tal-*clinic / senior medical officer* / XXXX / *polyclinic*

ASSISTANTE EN

PHARMACIE - RESTAURANT le *SMO* (médecin-chef) / je lui

ai dit / avec le *heating*

(chauffage) et comme ça / je

lui ai dit pas comme ces fous

que j'ai en bas je lui ai dit avec les appareils d'air conditionné allumés / il m'a dit *put down / a small tot of whisky* (mettez / une petite gorgée de whisky) avec le *coffee* (café) / c'est lui qui m'a donné pour mettre du whisky n'est-ce pas ? / *go ahead* (la permission)

Des différentes motivations que Romaine (1995) énumère pour expliquer la signification de l'alternance, celle qui correspond le mieux au comportement de cette locutrice lorsque l'alternance intraphrastique devient très fréquente dans son discours, c'est la pression culturelle qui la pousse à privilégier l'anglais pour montrer son appartenance à une élite. L'emploi de la langue extra-communautaire peut être vu comme l'expression linguistique du rejet d'un type de vie (ibid.). Romaine rend compte de l'étude de Gal (1979), qui atteste l'emploi de l'allemand par les femmes de la communauté hongrophone en Autriche comme un signe du rejet de la vie paysanne. Dans le cas de la locutrice maltaise, le choix de l'anglais pourrait signifier le rejet de l'identité insulaire, l'affirmation d'une distance voulue entre elle et ces «criards» à l'esprit borné, au niveau d'éducation inférieur. Parallèlement, elle affirme une mentalité plus ouverte, moderne, européenne. L'alternance est donc, chez cette locutrice, métaphorique, et constitue une affirmation d'appartenance à un groupe, en même temps que la stigmatisation d'un autre. Son comportement est aussi dans une certaine mesure interprétable dans la perspective du «language-crossing» («franchissement des langues», Rampton, 1995) qui rend compte de l'emploi par un locuteur d'un code associé à une ethnie autre que la sienne, par souci d'établir une certaine identité et des pratiques communes avec les membres de l'ethnie légitime, et/ou de souligner des divergences entre soi-même et une communauté particulière (ibid.). Pour revenir à l'idée de la mondialisation de l'anglais, ce type de recours à la langue démasque l'illusion que l'anglais favorise la communication unifiée : l'anglais se révèle être lié à l'inégalité et entrave dans une certaine mesure la communication, parce qu'il peut servir d'outil d'exclusion de certaines catégories d'individus, 
ne fût-ce que symboliquement, en dehors de l'empire culturel qui lui est associé (Pennycook, 2005). L'anglais se révèle être une clé de l'accès au pouvoir (Shelton, 2007).

La transaction de l'assistante en pharmacie dans un bureau de la compagnie financière reflète encore plus clairement les significations culturelles de l'alternance. L'anglais (ou plus précisément le MMA), semble être la L1 de l'agent financier (L1), qui accueille l'assistante en pharmacie (L2) et sa sœur (L5). L2 tâche de se montrer à la hauteur du choix linguistique de l'agent en employant elle aussi le MMA; par cette accommodation linguistique, la locutrice veut manifester des compétences d'anglais et par là le fait de posséder le même bagage social que son interlocuteur :

L1 : eqq da $=$ kien $/ *$ three five nine four* il-*holding* kien / *so if it was split up / between how much four?*

\section{L2 : *four / and my mother*}

L5 : * *and my mother* / ehe

L1 : *five* / i- i- imma

1-*mother* użufruttwarja?

L2 : $\quad$ *my mother* tieћu nofs / *my mother takes half*

L1 : $\quad$ in-nofs

L2 : u aћna *the other half we divide in four*

ASSISTANTE EN

PHARMACIE - COMPAGNIE FINANCIÈRE
L1 : bon celui-ci c'était / *three five nine four* (trois cinq neuf quatre) la *holding* (participation) c'était / *so if it was split up / between how much four?* (alors s'il a été divisé / entre combien quatre?)

L2 : *four / and my mother* (quatre / et ma mère)

L5 : $\quad$ *and my mother* (et ma mère) / oui

L1 : *five* (cinq) / m- m- mais la *mother* (mère) est-elle usufruitière?

L2 : $\quad$ *my mother* (ma mère) prend la moitié / *my mother takes half* (ma mère prend la moitié)

\section{L1 : $\quad$ la moitié}

L2 : et nous *the other half we divide in four* (l'autre moitié nous la divisons en quatre)

La traduction immédiate d'un énoncé maltais en anglais semble indiquer de sa part un effort conscient pour utiliser l'anglais autant que possible [*my mother* tiehu nofs / *my mother takes half* (*my 
mother* (ma mère) prend la moitié / *my mother takes half* (ma mère prend la moitié)]. Le fait que ce choix de code soit déterminé par la formalité de la situation, par l'identité de l'interlocuteur qu'elle respecte, le contexte spatial du bureau, le sujet technique de la transaction et la relation inégale entre elle et son interlocuteur expert, est rendu évident lorsqu'on observe un comportement très différent de la même locutrice dans une situation informelle.

Dans le texte DINER en effet, la sœur de l'assistante en pharmacie et sa famille sont invitées à diner chez elle, son mari le professeur et leur fils de quinze ans. L'assistante en pharmacie parle en maltais à sa famille lorsqu'elle est plus décontractée; elle se construit alors une identité de simple membre d'une famille qui, d'une manière générale, parle maltais comme langue dominante, par exemple lorsqu'elle taquine son beau-frère (L4) en omettant certains phonèmes, signe d'informalité :

L3 : < à son beau-frère > all=ra m'intix taghmel kif qallek il-XX? < à propos d'un médecin que $\mathrm{L} 4$ a rencontré à la clinique où travaille L3 (l'assistante en pharmacie) et qui a proposé à L4 de mettre un stylo sur le front pour ses fréquents maux de tête $>$ / kemm dhaqt

L4 : dak il-hin bdejt

L3 : kemm XXX / ghidtilhom $<$ ses collègues $>$ min gћandu wgigћ ta' ras? gћidtilhom ejja $\mathrm{X}$ bil-bajrow / 1-ghada reġa' rani / ma nafx kif gie l-ghada / XXXX

L4: XXX bajrow?

L3 : u regia' qalli:

L4 : qalli (nixrob tadam=?) ghallallergija hu?

L3 : ghall-allergija // haw= ghand= ikun / ghall-ugigh ta' ras xi mkien haw $=<$ rires $>$

ASSISTANTE EN

PHARMACIE - DINER
L3 : alors tu ne fais pas comme le XX t'a dit? / j'en ai tellement ri

L4 : à ce moment-là j'ai commencé

L3 : XXX tellement beaucoup / je leur ai dit < à ses collègues $>$ qui a mal à la tête? je leur ai dit allez $\mathrm{X}$ avec un stylo / le lendemain il m'a vue encore / je ne sais pas pourquoi il est revenu le lendemain / $\underline{\mathrm{XXXX}}$

L4 : XXX stylo?

L3 : et il m'a encore dit:

L4 : il m'a dit de boire du jus de tomates pour l'allergie n'est-ce pas?

L3 : pour l'allergie // là je crois / pour les maux de tête quelque part là < rires > 
Une fois les convives installés à table, pourtant, on remarque chez elle un changement de footing, reflété dans l'usage d'un style moins relâché. La cause est sans doute attribuable en partie au cadre spatial, la salle à manger des hôtes étant imposante, avec ses meubles et ornements précieux, et en partie au sujet, lorsque la maitresse de maison parle de thèmes qui affirment son statut bourgeois, comme sa bonne (exemple 13), les études de son fils, son expertise dans le monde médical et ses voyages à l'étranger. Dans tous ces cas, l'assistante en pharmacie emploie le MMA. Ainsi, les noms des jours de la semaine sont produits en anglais, conformément à ce qui est devenu une mode reflétant une certaine affectation chez un nombre considérable de maltophones.

\section{L2 : (kellek il-*maid* illum?)}

L3 : is-soltu nghidilha tigi

*Tuesday* / imm= aћna m'aћniex daqshekk *fixed* ta / naghtu kas nghidilha / gieli tghid / tghidli tista * Monday* jow *Wednesday* inti?

ASSISTANTE EN

PHARMACIE - DINER
L2 : tu avais la *maid* (bonne) aujourd'hui?

L3 : d'habitude je lui dis de venir le *Tuesday* (mardi) / mais nous le jour c'est pas tellement $*$ fixed $*$ (fixe) tu sais / par exemple je lui dis / parfois elle dit / elle me dit tu peux *Monday* (lundi) ou $*$ Wednesday* (mercredi) tu crois?

Rampton (1995) problématise l'ethnicité et la socialisation avec une mise en relief de l'idée de parenté biologique et d'héritage par rapport à l'ethnie, chez des adolescents engagés dans l'interaction multiethnique ${ }^{20}$ dans des établissements scolaires britanniques, où le franchissement des langues implique la conscience d'un mouvement distinct au-delà de frontières sociales. Le locuteur aurait pour but d'adopter l'ethnicité d'une communauté de pairs qui n'est pas la sienne, ou de se joindre à cette communauté en créant une nouvelle ethnicité. Dans le cadre de notre étude, l'appartenance à divers groupes ethniques comme point de départ n'est pas fondamentalement pertinente, les locuteurs ayant tous, à première vue, la même origine maltaise. Pourtant, le comportement de locuteurs comme l'assistante en pharmacie nous amène à réfuter l'idée

20. Les réseaux principaux sont, dans l'étude de Rampton : les groupes indien, pakistanais, noir, anglais de souche, bangladais et italien. 
d'une ethnicité stable, unique et universellement partagée par la population, et à embrasser une définition de l'ethnicité telle que celle fournie par Le Page et Tabouret-Keller (1985), que Rampton interprète ainsi : l'ethnicité implique généralement la combinaison d'éléments tels que la conscience d'un lieu, d'une origine et d'une destinée communs, le partage culturel et/ou linguistique, un certain degré de consensus sur l'évaluation des non-membres en tant que «autres», l'identification avec le groupe, et sa reconnaissance par les non-membres (Rampton, 1995, p. 487). Nos locuteurs franchissent les frontières entre styles et parfois même entre codes - maltais (formel et informel), MMA - lorsqu'ils sont amenés à communiquer dans des situations marquées par différents degrés et différents types de formalité. Ce faisant, ils montrent dans certaines occasions ${ }^{21}$ la volonté d'appartenir à un groupe plutôt qu'à un autre, de souligner la différence (plutôt que le partage des normes) et une destinée distincte de celle du groupe linguistiquement majoritaire (les locuteurs de maltais L1). Ils se placent dans un groupe considéré comme celui des locuteurs privilégiés d'un code plus prestigieux (les locuteurs du MMA).

Rampton (1995) montre que les individus négocient une variété d'identités interactionnelles et institutionnelles, où les codes (dans notre cas, nous pouvons aussi ajouter les styles) sont porteurs d'ensembles complexes de connotations symboliques. Le franchissement des frontières de langues soulève le problème de la légitimité : l'assistante en pharmacie doit à certains moments faire de réels efforts pour se conformer aux attentes d'alternance qu'elle ressent comme s'imposant à elle dans l'interaction formelle et technique avec l'un des propriétaires de la compagnie financière.

L'identité sociale, tout comme l'appartenance ethnique, n'est pas fixe, mais est négociée au fur et à mesure que l'interaction se déroule : selon Rampton, l'ethnicité est une construction par laquelle les locuteurs créent des contrastes et se positionnent, en créant, en exprimant et en interprétant des distinctions sociales. L'identité, par exemple de classe ou d'ethnie, devient pertinente tout en changeant à différents moments de l'interaction, selon les besoins et les pressions situationnelles. Elle tire sa signification de la nature des interactions particulières dans lesquelles elle est activée (Rampton, 1995, p. 486).

21. Il en va ainsi, par exemple, de l'assistante en pharmacie lorsqu'elle interagit avec son supérieur à la clinique, ou avec l'agent financier. 
Nous interprétons le comportement des locuteurs des classes moyenne et dirigeante, qui font preuve de plus en plus d'instances d'alternance dans leur discours, comme un reflet des changements culturels qui altèrent la conscience ethnique de cette catégorie de locuteurs. Les linguistes et historiens de la culture (Borg \& Mifsud, 2005; Cassar, 2001; Ebejer, 1989) identifient trois piliers ayant traditionnellement régi et affermi l'identité maltaise : la religion catholique, la langue maltaise et, ironiquement, la présence de pouvoirs étrangers qui consolidait la cohésion du peuple face aux dominations diverses. On peut donc se demander dans quelle mesure les attitudes linguistiques de dissociation ethnique et de distinction psychologique seraient attribuables au bouleversement social qui a porté atteinte à chacun comme à la fusion de ces trois éléments, autrefois facteurs unificateurs. Parmi les forces qui semblent sonner le glas de la cohésion ethnique autour des facteurs identitaires traditionnels, les principaux responsables sont sans doute l'enseignement généralisé de l'anglais, la portée moindre de la religion dans la société et, paradoxalement, la cessation des dominations étrangères. Il semble que ces changements rapides soient en train d'affaiblir le tissu communautaire et font perdre les marques d'appartenance autour de points de repère communément partagés; ils favorisent par contre l'émergence de nouveaux modèles.

Un comportement similaire à celui de l'assistante en pharmacie visà-vis de l'anglais est manifesté par le cadre, second locuteur non professionnel de la parole publique. Une session de planification du travail entre le cadre et son supérieur, collègues dans une société de développement de logiciel, montre le recours fréquent à des termes techniques en anglais, sans doute provoqué par le contexte de technologie avancée qui caractérise leur profession. Les termes techniques sont accompagnés d'autres termes communément réalisés en anglais, comme les noms des mois et les termes de gestion (handed over, transition, by end of August; exemple 14) :

\author{
L1 : [...] imbagћad gћandna \\ $1-*$ instant pricing / phase two / \\ instant pricing phase two* qed \\ narawna / li aћna ha nlestu \\ 1-analiżi / u t-*technical specs* / \\ imma *development* jista' jigi \\ *handed over* ukoll / imma: /
}

\author{
L1 : [...] et puis on a la *instant \\ pricing / phase two / instant \\ pricing phase two* (facturation \\ instantanée / phase deux / la \\ facturation instantanée phase \\ deux) nous nous voyons / que \\ nous on aura terminé l'analyse /
}




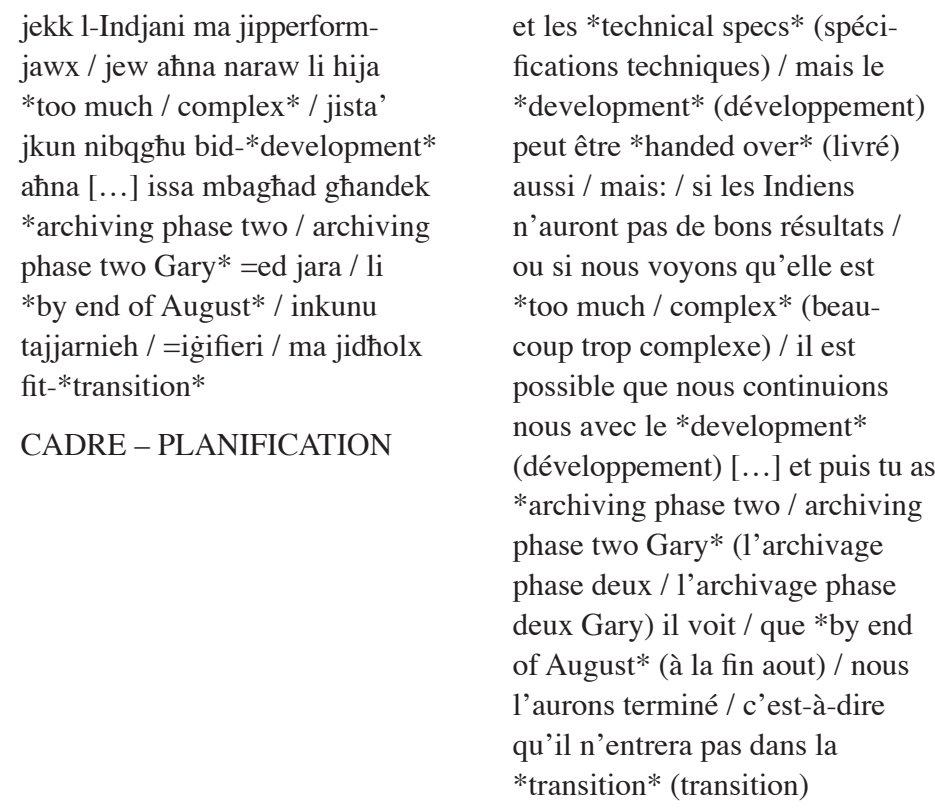

Ailleurs chez ce locuteur (L2), même si l'interlocuteur est toujours son supérieur (L1), dans une interaction informelle du fait du ton humoristique et du sujet trivial (l'organisation d'une fête de décernement de prix), le recours à l'anglais est minimal. L'identité et l'efficacité professionnelles n'y constituent plus un enjeu :

L1 : *Antoine* $<$ l'un des dirigeants de l'entreprise $>$ kellmek jew?

L2 : *Antoine* $=$ ed nitkellem miegћu [...] le rrispondejtu / ghidtlu: // inti: ibda filkas itlaq baxx baxx hafna ћa jagћmlu hekk ta / jien per eżempju rrid nitlaq naq=a kmieni wkoll / irrid nitlaq / ghas-sitta w nofs / minn tal-inqas

L1 : GHAS-sitta w nofs?

L2 : ehe

L1 : > railleur, fait une grimace >
L1 : *Antoine* il t'a parlé quoi?

L2 : *Antoine* je suis en contact avec lui [...] non je lui ai répondu / je lui ai dit: // toi: tu commences et puis si tu veux tu peux partir en catimini y en a plein qui feront comme ça / moi par exemple je dois partir un peu tôt aussi / je dois partir / vers six heures et demie / au moins

L1 : VERS six heures et demie?

L2 : ouais

L1 : < railleur, fait une grimace > 


\section{L2 : ehe / u le jista' jkun ma ddumx daqshekk}

L1 : eh / jien gћadni gћadni ma rqadtx ta llum?

L2 : le tidher =igifieri imma / $\mathrm{t}=\mathrm{idx}$ nibdew mela ma jmurx torqodli haw $=$ ?

CADRE - PRIX
L2 : ouais / mais non il est possible qu'elle dure pas si longtemps

L1 : hein / tu sais que j'ai pas encore dormi aujourd'hui?

L2 : ben c'est clair mais / tu veux qu'on commence avant que tu t'endormes?

Le recours aux différents codes parait donc revêtir des implications stylistiques empreintes de valeurs sociolinguistiques particulières : respect de contraintes formelles, volonté de se rapprocher ou de se distancier d'un groupe, affichage du statut social, de l'efficacité et de l'autorité professionnelle, ou détente lorsqu'on interagit au sein de l'intimité familiale.

\section{Conclusion}

Au terme de cette discussion, il est clair que diverses sources d'influence opèrent ensemble pour forger la configuration particulière du bilinguisme à Malte. Il en résulte des tendances conflictuelles au niveau des attitudes et préférences linguistiques. Tant l'anglais que le maltais figurent dans des contextes formels, mais il semble que seuls les contextes institutionnels agissent comme des barrières plus ou moins solides contre l'anglais. Les contextes formels non strictement protocolaires, gérés par les locuteurs parce que se situant au-delà des sphères soumises aux impositions linguistiques officielles, ouvrent la voie à l'emploi parfois même affiché de l'anglais, à différents degrés de présence (le taux d'anglais dans les séquences en MMA étant variable). La facilité et la fréquence des passages intraphrastiques d'une langue à l'autre témoignent des frontières codiques floues dans ces contextes et dans les contextes d'informalité.

Alors que la recherche en translanguaging accentue cette idée de souplesse qui permet aux locuteurs plurilingues de recourir à leur répertoire linguistique en alternant avec une aisance naturelle pour produire du discours sémantiquement cohérent, le comportement verbal des locuteurs professionnels de la parole publique dans notre corpus démontre que les cadres officiels ont des retombées sur le degré de fluidité avec lequel on pourrait passer d'une langue à l'autre. Le mouvement à l'intérieur du répertoire des locuteurs devient plus rigide dans 
les situations protocolaires à cause des contraintes de monolinguisme qui exercent des pressions bien réelles. C'est dans les contextes non institutionnels, où les locuteurs réalisent fortuitement l'alternance pour des propos tout à fait spontanés, que les frontières entre langues deviennent plus perméables.

Le corpus confirme la réalité d'un phénomène linguistique commun parmi les membres de la classe moyenne, qui consiste à privilégier les termes anglais pour exprimer quelques notions comme les jours de la semaine, les dates, etc. C'est ce genre de termes anglais, à part le jargon technique, qui continue d'avoir quelque présence, même si très diminuée, à l'intérieur du discours produit en maltais langue dominante dans des contextes protocolaires.

En dehors des contextes institutionnels, l'anglais, perçu comme langue de prestige, connote le statut social, l'autorité et/ou l'efficacité professionnelles. Ces tendances vont dans le même sens que la théorie sur l'impact de l'anglais comme moteur et conséquence de la mondialisation : l'anglais peut servir d'arme, une arme par laquelle le locuteur cherche à «se tailler la part du lion» dans l'appropriation du pouvoir social. Des pressions culturelles peuvent en effet pousser le locuteur à privilégier l'anglais à travers l'emploi du MMA : il afficherait ainsi son appartenance à l'élite, tout en se distanciant d'un groupe stigmatisé. En franchissant les frontières des langues et par là des styles, les locuteurs montrent leur désir et leur ambition de se forger des identités particulières, institutionnellement, socialement, et selon les traits caractéristiques de chaque interaction. L'emploi de tel ou tel style renvoie au problème de la légitimité d'appartenance : on parle comme un groupe particulier parce qu'on souhaite en être considéré comme membre à part entière.

Le comportement linguistique des classes moyenne et dirigeante, très souvent marqué par l'alternance, semble être la conséquence de changements culturels modernes survenus à Malte, auxquels ces classes sont sensibles. Les facteurs culturels qui assuraient autrefois l'union sociale cèdent la place à des facteurs susceptibles de produire la fragmentation de la société maltaise en groupes linguistiquement distincts. 


\section{RÉFÉRENCES BIBLIOGRAPHIQUES}

Baker, Colin, Jones, Bryn \& Lewis, Gwyn. (2012). Translanguaging: Origins and Development from School to Street and beyond. Educational Research and Evaluation, 18(7), 641-654.

Bell, Allan. (1984). Language Style as Audience Design. Language in Society, 13, 145-204.

Bell, Allan. (2001). Back in Style: Reworking Audience Design. Dans P. Eckert \& J. R. Rickford (dir.), Style and Sociolinguistic Variation (p. 139-169). Cambridge : CUP.

Bilger, Mireille \& CAppeau, Paul. (2004). L'oral ou la multiplication des styles. Langage et société, 3(109), 13-30.

Borg, Albert. (1980). Language and Socialization in Developing Malta. Work in Progress, 13, Department of Linguistics, Université d'Édimbourg, 60-71.

Borg, Albert. (1988). Ilsienna: Studju Grammatikali. Malte : Has-Sajjied.

Borg, Albert \& Mifsud, Manwel. (2005). Il-pożizzjoni tal-Malti f'Malta. Il-Malti, 77, 6-16.

Busuttil Bezzina, Anne-Marie. (2013). La variation stylistique en maltais : étude des usages concrets de la langue appuyée sur une approche contrastive des phénomènes variationnels en maltais et en français (Thèse de doctorat en sciences du langage inédite). Université Paris Ouest - Nanterre et Université de Malte.

CAmilleri, Antoinette. (1995). Bilingualism in Education. Heidelberg : Julius Groos Verlag.

Canagarajah, A. Suresh. (1999). Resisting Linguistic Imperialism in English Teaching. Oxford : OUP.

Caruana, Sandro. (2011). Bilingualism and Language Policy in Malta. Dans P. Ramat \& E. Miola (dir.), Language Contact and Language Decay: Socio-Political and Linguistic Perspectives (p. 11-46). Pavie : IUSS.

CAssar, Carmel. (2001). Malta: Language, Literacy and Identity in a Mediterranean Island Society. National Identities, 3(3), 257-275.

Coupland, Nikolas. (2001). Language, Situation, and the Relational Self: Theorizing Dialect-style in Sociolinguistics. Dans P. Eckert, J. R. Rickford (dir.), Style and Sociolinguistic Variation (p. 185-210). Cambridge : CUP.

Cucciardi, Mario. (1990). A Sociolinguistic Study of Numerical Expressions in Malta (Mémoire de Licence d'Éducation inédit). Université de Malte.

EBEJER, Francis. (1989). The Bilingual Writer as Janus. Malte : Foundation for International Studies. 
ECKert, Penelope. (2000). Linguistic Variation as Social Practice. Oxford : Blackwell.

Gadet, Françoise. (1998). Cette dimension de variation que l'on ne sait nommer. Sociolinguistica, 12, 53-71.

Gadet, Françoise. (2005). Research on Sociolinguistic Style. Dans U. Ammon et coll. (dir.), Sociolinguistics/Soziolinguistik (p. 1353-1360). Berlin et New York : Mouton de Gruyter.

GADET, Françoise. (2007). La variation sociale en français. Paris : Ophrys. Gal, Susan. (1979). Language Shift. Social Determinants of Linguistic Change in Bilingual Austria. New York : Academic Press.

Garcia, Ofelia. (2009). Education, Multilingualism and Translanguaging in the 21st century. Dans A. K. Mohanty et coll. (dir.), Multilingual Education for Social Justice: Globalising the Local (p. 140-158). New Delhi : Orient BlackSwan.

Goffman, Erving. (1981). Footing. Dans E. Goffman, Forms of Talk (p. 124159). Philadelphie : University of Pennsylvania Press.

Gumperz, John J. (1982). Discourse Strategies. Cambridge : CUP.

Labov, William. (1972). Sociolinguistic Patterns. Philadelphie : University of Pennsylvania Press.

Le Page, Robert \& Tabouret-Keller, Andrée. (1985). Acts of Identity. Cambridge : CUP.

Pennycook, Alastair. (2005). Global and Worldly Englishes: Discommunities and Subcultural Empires. Article présenté à l'Institute of European Studies, Berkeley, CA.

REHORICK, Sally. (2009). Le rôle crucial du système d'éducation pour créer des citoyens bilingues : le Nouveau-Brunswick comme microcosme du Canada. Disponible sur <www.ocol.gc.ca/html/rehorick_f.php $>$.

Rampton, Ben. (1995). Language Crossing and the Problematisation of Ethnicity and Socialisation. Pragmatics, 5(4), 485-513.

Romaine, Suzanne. (1995). Bilingualism (2 éd.). Malden-Oxford : OUP.

Schilling-Estes, Natalie. (2002). Investigating Stylistic Variation. Dans J. K. Chambers, P. Trudgill \& N. Schilling-Estes (dir.), Language Variation and Change (p. 375-401). Oxford : Blackwell.

Shelton, Linda. (2007). Importing Canagarajah's Global English Theories. English Teaching: Practice and Critique, 6(2), 58-71.

TAnnen, Deborah. (2005). Conversational Style: Analyzing Talk among

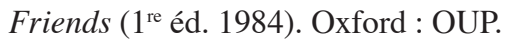

Traugott, Elizabeth C. \& Romaine, Suzanne. (1985). Some Questions for the Definition of 'Style' in Socio-Historical Linguistics. Folia Linguistica Historica, VI, 7-39. 


\section{ANNEXE}

\begin{tabular}{|l|l|}
\hline \multicolumn{2}{|c|}{ Conventions de transcription } \\
\hline /, //, // & pause - brève, moyenne, longue \\
MAJUSCULES & mot(s) mis en relief par une intensité accrue \\
$=$ & phonème(s) non prononcé(s) \\
$* \ldots *$ & début et fin de l'alternance \\
$:$ & allongement de la voyelle \\
\hline
\end{tabular}

